\title{
Generating 5-regular planar graphs
}

\author{
Guoli Ding * \\ Department of Mathematics, Louisiana State University \\ Baton Rouge, Louisiana 70803, USA \\ ding@math.lsu.edu \\ Jinko Kanno † Jianning Su \\ Mathematics and Statistics Program, Louisiana Tech University \\ Ruston, Louisiana 71272, USA \\ jkanno@latech.edu,jsu007@latech.edu
}

\begin{abstract}
For $k=0,1,2,3,4,5$, let $\mathcal{P}_{k}$ be the class of $k$-edge-connected 5 regular planar graphs. In this paper, graph operations are introduced that generate all graphs in each $\mathcal{P}_{k}$.
\end{abstract}

\footnotetext{
${ }^{*}$ Research partially supported by NSF grants DMS-0556091 and ITR-0326387.

${ }^{\dagger}$ Research partially supported by Board of Regents grant LEQSF(2004-07)-RD-A-22.
} 


\section{Introduction}

A well known theorem of Steinitz and Rademacher [15] states that the class $\mathcal{G}$ of 3-connected 3-regular simple planar graphs can be generated from the tetrahedron by adding handles (also known as ears), a graph operation which is illustrated in Figure 1.1 below.
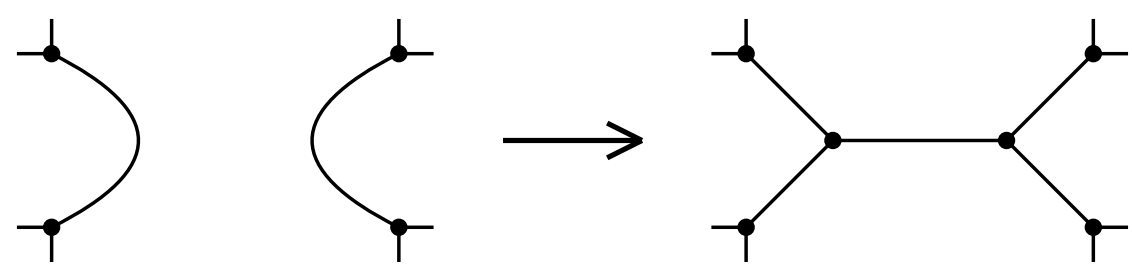

Figure 1.1: Adding a handle.

This result can be stated more precisely as follows. For every graph $G$ in $\mathcal{G}$, there is a sequence $G_{0}, G_{1}, \ldots, G_{t}$ of members of $\mathcal{G}$ such that $G_{0}$ is the tetrahedron, $G_{t}$ is $G$, and each $G_{i+1}$ is obtained from $G_{i}$ by adding a handle. In $[1,2,4,12]$, analogous results are obtained for 3-regular simple planar graphs with other connectivities. For 4-regular simple planar graphs, the situation is similar and the readers are referred to $[3,9,10]$. In this paper, we will consider 5 -regular planar (not necessarily simple) graphs.

For $k=0,1,2,3,4,5$ let $\mathcal{P}_{k}$ be the class of $k$-edge-connected 5 -regular planar graphs. We introduce, for each $\mathcal{P}_{k}$, a set of graph operations for which all graphs in $\mathcal{P}_{k}$ can be generated from the smallest members of $\mathcal{P}_{k}$ by these operations. Like the operation of adding a handle, these operations only modify a graph locally. In fact, each of these operations only changes the number of vertices by either two or four. There are twelve operations in total involved in, and most of them are needed for only one $\mathcal{P}_{k}$. In the remaining six sections of this paper, we consider the six different connectivities separately. In some sections, we also discuss the corresponding results for simple graphs.

We emphasize that planarity is not essential in our theorems; our theorems work for all surfaces. Let $\Sigma$ be a surface and $\mathcal{G}_{k}$ be the class of $k$-edge-connected 5 -regular graphs that can be drawn on $\Sigma$. Then $\mathcal{P}_{k}$ can always be substituted by $\mathcal{G}_{k}$, in the statements and the proofs of our theorems. As a consequence, such a substitution is also valid if $\mathcal{G}_{k}$ is the class of 
all $k$-edge-connected 5 -regular graphs. We choose to only deal with planar graphs because this is the most interesting class of graphs, the generalizations do not have any new ideas, and we do not need to introduce more terminology.

We should also point out that, in the rest of the discussions, instead of trying to generate all graphs in $\mathcal{P}_{k}$, we shall consider how to reduce a graph in $\mathcal{P}_{k}$ into a smaller graph in $\mathcal{P}_{k}$. this is an equivalent problem but it is more convenient to work with. To be more precise, we say that a graph $G$ can be reduced within a class $\mathcal{G}$ of graphs by a set $G_{0}=G, G_{t}=H$, and each $G_{i}$ is obtained from $G_{i-1}$ by applying an operation in $\mathcal{O}$ only once. The set of operations will be made clear later in this paper.

We close this section by introducing some terminology and notation to be used in this paper. Let $5 K_{2}, 3 K_{2}^{L}$, and $K_{2}^{2 L}$ be the only 5 -regular graphs on two vertices with 0,2 , and 4 loops, respectively. If $Z$ is a vertex, an edge, or a set of vertices or edges of a graph $G$, then we denote by $G \backslash Z$ the graph obtained from $G$ by deleting $Z$. Let $G$ be a plane graph, that is, a planar drawing of a planar graph. If a vertex $x$ of $G$ has degree four, then by splitting $x$ we mean the operation illustrated in Figure 1.2. Observe that, when $G$ and $x$ are given, there are two possible outcomes of this operation since there are two ways to pair the four edges. Clearly, an edge could be paired with itself, if it is a loop. In fact, it is easy to verify that, if $x$ is incident with a loop $e$, then splitting $x$ has only one outcome - it is the graph obtained from $G \backslash e$ by contracting an edge incident with $x$.

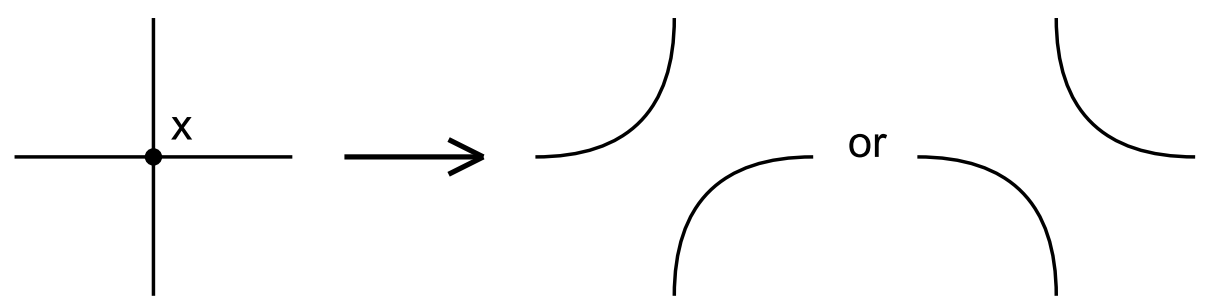

Figure 1.2: Splitting a vertex $x$.

\section{The class of all 5-regular planar graphs}

We start with the definitions of the three graph operations that are used to generate all graphs in $\mathcal{P}_{0}$. Our first operation is an analog of "removing a 
handle", the inverse of adding a handle.

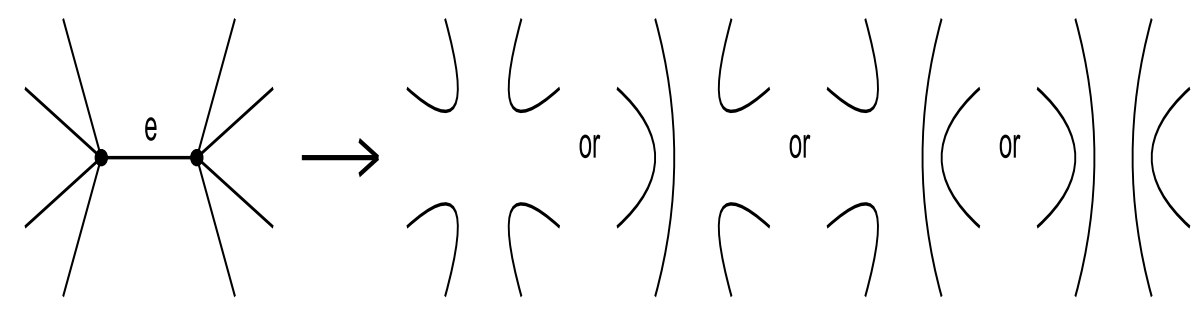

Figure 2.1: Operation $\mathcal{O}_{1}$.

Here, $e$ is assumed to be simple. That is, it is not a loop and it is not in parallel with any other edges. Clearly, applying $\mathcal{O}_{1}$ to $e$ is equivalent to deleting $e$ and then splitting the end vertices of $e$. Since there are up to two ways to split each end of $e$, there are up to four different outcomes when $\mathcal{O}_{1}$ is applied. We need to point out that $\mathcal{O}_{1}$ is very special among the twelve operations we introduce in this paper. It is one of the only two operations (the other one is $\mathcal{O}_{9}$ which will be defined in Section 6) that depend on the drawing of the graph and it is the only one that is needed to generate graphs in every $\mathcal{P}_{k}$.
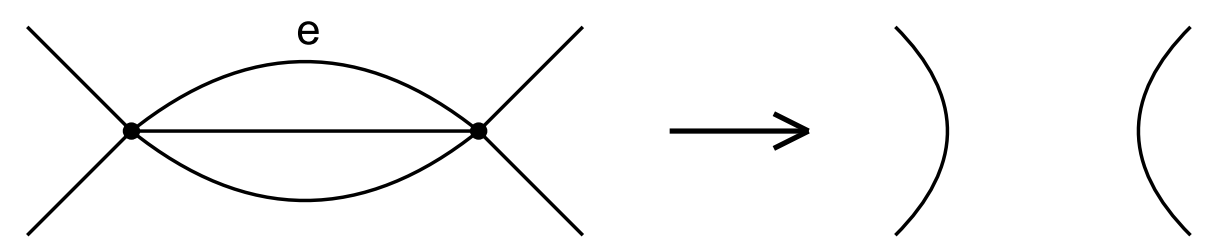

Figure 2.2: Operation $\mathcal{O}_{2}$.

In the definition of $\mathcal{O}_{2}$, the edge $e$ is in a parallel family of size exactly three. This is another analog of removing handles. Like in the operation of splitting a vertex, if a loop is involved in, it is actually being deleted when $\mathrm{O}_{2}$ is applied to $e$.

The definition of $\mathcal{O}_{3}$ is quite clear from Figure 2.3. It is worth mentioning that $\mathcal{O}_{3}$ preserves the edge-connectivity of a graph. This is because $\mathcal{O}_{3}$ is the result of contracting five edges and contracting edges does not change 


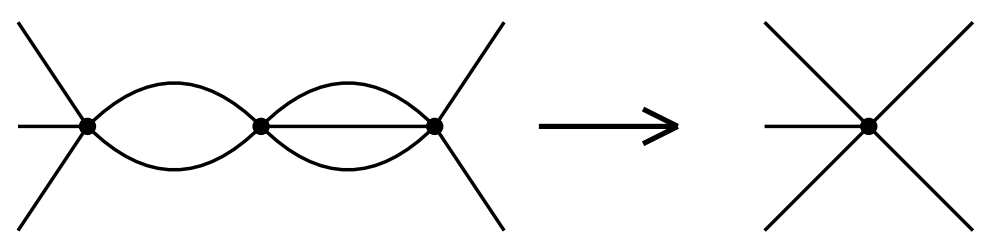

Figure 2.3: Operation $\mathcal{O}_{3}$.

edge-connectivity. The operations $\mathcal{O}_{1}, \mathcal{O}_{2}$, and $\mathcal{O}_{3}$ are reducing the order and size of the graph by two and five, respectively.

The main results in this section are easy to prove. We choose to include them here for the completeness of this paper. Another reason for including them here is that the lemmas that are used to prove these results will be needed in later sections as well.

Theorem 2.1 Every graph in $\mathcal{P}_{0}$ can be reduced within $\mathcal{P}_{0}$ by $\mathcal{O}_{1}$ and $\mathcal{O}_{3}$ to a graph for which every component is $5 K_{2}, 3 K_{2}^{L}$ or $K_{2}^{2 L}$.

This result is an obvious consequence of the following observation. A graph $G$ is called an alternating path if it has vertices $x_{1}, x_{2}, \ldots x_{2 t}$, where $t \geq 1$, such that there are three parallel edges from $x_{2 i-1}$ to $x_{2 i}(i=1,2, \ldots, t)$ and two parallel edges from $x_{2 i}$ to $x_{2 i+1}(i=1,2, \ldots, i-1)$. If we add two more edges from $x_{1}$ to $x_{2 t}$, then the resulting graph is called an alternating cycle. Notice that an alternating cycle is 5-regular, and an alternating path is "almost" 5-regular - other than the two ends, all its vertices have degree five.

Lemma 2.1 Let $G$ be 5-regular and connected. If $G$ has no simple edges, then $G$ is either an alternating cycle or an alternating path with a loop at each of its ends.

Proof. Let $H$ be the graph obtained from $G$ by deleting all loops and then deleting all but one edge from each parallel family. Clearly, the maximum degree of $H$ is at most two and thus $H$ is either a cycle or a path. If $H$ has only two vertices, then $G$ is either the alternating path $3 K_{2}^{L}$ or the alternating cycle $5 K_{2}$. If $H$ has more than two vertices, then $G$ is an alternating cycle when $H$ is a cycle and $G$ is an alternating path, plus two 
loops, when $H$ is a path.

Proof of Theorem 2.1. Notice that the result of applying $\mathcal{O}_{1}$ or $\mathcal{O}_{3}$ to a graph in $\mathcal{P}_{0}$ remains in $\mathcal{P}_{0}$. Thus we only need to show that, if $G \in \mathcal{P}_{0}$ has a component $C$ with more than two vertices, then either $\mathcal{O}_{1}$ or $\mathcal{O}_{3}$ can be applied to $G$. But this is clear: if $C$ has a simple edge, then $\mathcal{O}_{1}$ can be applied; if $C$ has no simple edges, then, by Lemma 2.1, $\mathcal{O}_{3}$ can be applied.

Lemma 2.2 Let $G$ be a connected graph in $\mathcal{P}_{0}$. Suppose $G$ is not $5 K_{2}$ or the null graph. Then either $\mathcal{O}_{1}$ or $\mathcal{O}_{2}$ can be applied to $G$ such that $5 K_{2}$ is not a component of the resulting graph.

Proof. By Lemma 2.1, we may assume that $G$ has a simple edge $e=x y$. Let $H$ be the result when $\mathcal{O}_{1}$ is applied to $e$. Clearly, we may assume that a component $C$ of $H$ is $5 K_{2}$, because otherwise we are done. Let $u, v$ be the two vertices of $C$. We consider the following four cases.

Case 1. At least four edges of $C$ are new. Then it is easy to see that exactly four edges of $C$ are new, $G$ has only four vertices, and $E(G)=$ $\{u v, u x, u x, u y, u y, v x, v x, v y, v y, x y\}$. In this case, let us change the pairing at $x$ from $\{u x, v x\}-\{u x, v x\}$ to $\{u x, u x\}-\{v x, v x\}$, then the result of applying $\mathcal{O}_{1}$ to $x y$ will be $3 K_{2}^{L}$, instead of $5 K_{2}$.

Case 2. Exactly three edges of $C$ are new. Then $E(G) \supseteq\{u v, u v, u x, u x$, $u y, v x, v x, v y, x y\}$. Clearly, either $y$ is incident with a loop or $y$ is incident with other two distinct edges. Like in the previous case, let us change the pairing of edges at both $x$ and $y$. Then it is easy to see that applying $\mathcal{O}_{1}$ to $x y$, with respect to the new pairing, result in a graph $H^{\prime}$ such that $H^{\prime}$ is connected and $H^{\prime}$ also has a loop. Thus $5 K_{2}$ is not a component of $H^{\prime}$, as required.

Case 3. Exactly two edges of $C$ are new. Then $E(G) \supseteq\{u v, u v, u v, u x$, $u x, v x, v x, x y\}$ or $E(G) \supseteq\{u v, u v, u v, u x, u y, v x, v y, x y\}$. Let us apply $\mathcal{O}_{2}$ to $u v$ and let $H^{\prime}$ be the resulting graph. In the former case, it is easy to see that $H^{\prime}$ is connected and $H^{\prime}$ has a loop. In the later case, it is also easy to see that $H^{\prime}$ is connected, and either $H^{\prime}=3 K_{2}^{L}$ or $H^{\prime}$ has more than two vertices. In all cases, $5 K_{2}$ is not a component of $H^{\prime}$, as required.

Case 4. Exactly one edge of $C$ is new. Then $E(G) \supseteq\{u v, u v, u v, u v, u x$, $v x, x y\}$. In this case, we apply $\mathcal{O}_{1}$ to $u x$. At $x$, we choose the pairing such that $v x$ is not paired with $x y$. Let $H^{\prime}$ be the resulting graph. Clearly, the component that contains $v$ can not be $5 K_{2}$ since it has loops. In case $H^{\prime}$ is disconnected, its only other component is the one that contains $y$. Suppose 
this component is $5 K_{2}$. Consider that there is precisely one new edge for this $5 K_{2}$ (otherwise, we can use Case 1, Case 2, or Case 3). Change the pairing at $x$, the resulting graph is connected and has loops. Therefore, $5 K_{2}$ is not a component of the resulting graph, as required.

\section{Connected 5-regular planar graphs}

The following is another graph operation which is needed to generate all graphs in $\mathcal{P}_{1}$.

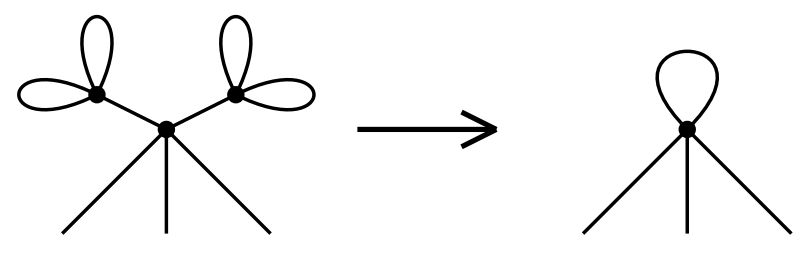

Figure 3.1: Operation $\mathcal{O}_{4}$.

The definition of $\mathcal{O}_{4}$ is quite clear from Figure 3.1. It is worth mentioning that $\mathcal{O}_{4}$ preserves 1-edge-connectivity.

Theorem 3.1 Every graph $G$ in $\mathcal{P}_{1}$ can be reduced within $\mathcal{P}_{1}$

(a) to $5 K_{2}, 3 K_{2}^{L}$ or $K_{2}^{2 L}$ by $\mathcal{O}_{1}, \mathcal{O}_{3}$, and $\mathcal{O}_{4}$; and

(b) to $3 K_{2}^{L}$ or $K_{2}^{2 L}$ by $\mathcal{O}_{1}, \mathcal{O}_{2}$, and $\mathcal{O}_{4}$, unless $G=5 K_{2}$.

To prove Theorem 3.1, we first prove two easy lemmas. An edge in a connected graph is a cut if its deletion disconnects the graph.

Lemma 3.1 Let $x$ be a degree-four vertex in a connected plane graph. Then at least one splitting at $x$ is connected, unless all the four edges that are incident with $x$ are cut edges.

Proof. Since $x$ has degree four, it is incident with four (not necessarily distinct) edges, say $e_{1}, e_{2}, e_{3}, e_{4}$, where $e_{i}=e_{j}$ for $i \neq j$ only if the edge is a 
loop. Let us assume that these four edges are listed in the same cyclic order as drawn on the plane around $x$. Suppose some $e_{i}$, say $e_{1}$, is not a cut edge. It follows that there is a cycle that contains both $e_{1}$ and $e_{i}$ for some $i \neq 1$. By symmetry, we assume that $i \neq 2$. Then it is easy to verify that the split $\left\{e_{1}, e_{2}\right\}-\left\{e_{3}, e_{4}\right\}$ results in a connected graph.

A vertex is singular if it is incident with three or more cut edges. Let $G$ be a graph in $\mathcal{P}_{1}$ and let $e=x_{1} x_{2}$ be a simple non-cut edge of $G$. We will call $e$ singular if each $x_{i}$ is incident with precisely two cut edges and $G \backslash\left\{x_{1}, x_{2}\right\}$ has precisely six components, Figure 3.2 illustrates what a singular edge should look like.

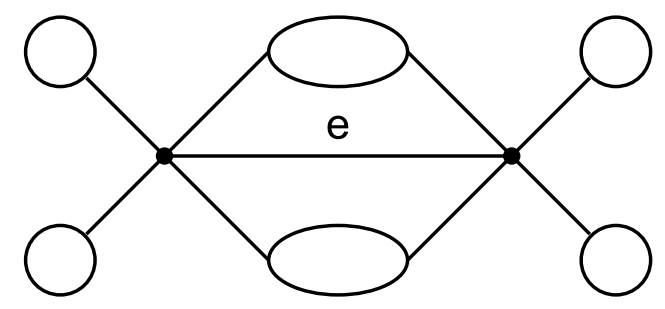

Figure 3.2: A singular edge $e$.

Lemma 3.2 Let $e=x_{1} x_{2}$ be a simple non-cut edge of a graph $G$ in $\mathcal{P}_{1}$. Suppose $x_{1}, x_{2}$ and $e$ are non-singular. Then at least one outcome of $\mathcal{O}_{1}$ on $e$ is connected.

Proof. First, $G \backslash e$ is connected since $e$ is not a cut. We also observe that each $x_{i}$ is incident with at most three cut edges of $G \backslash e$. Because otherwise, the four edges that are incident with $x_{i}$ in $G \backslash e$ must be all cut edges of $G \backslash e$. Thus, by putting $e$ back, we conclude that $x_{i}$ is incident with at least three cut edges of $G$, contradicting the assumption that $x_{i}$ is non-singular. By Lemma 3.1, $G \backslash e$ can be split at $x_{1}$ so that the resulting graph $H$ is still connected. If not all edges incident with $x_{2}$ in $H$ are cut edges of $H$, then Lemma 3.2 follows from the application of Lemma 3.1 to $H$ at $x_{2}$. Thus we may assume that all the four edges in $H$ that are incident with $x_{2}$ are cut edges of $H$. By considering the reverse operation of the splitting at $x_{1}$, we deduce that $x_{2}$ is incident with at least two cut edges of $G$. Since $x_{2}$ is not singular, it must be incident with precisely two cut edges of $G$. By symmetry, $x_{1}$ is also incident with precisely two cut edges of $G$. It follows that 
$G \backslash\left\{x_{1}, x_{2}\right\}$ has at least five components. Since $e$ is not singular, $G \backslash\left\{x_{1}, x_{2}\right\}$ can not have more than five components. Hence $G \backslash\left\{x_{1}, x_{2}\right\}$ has exactly five components. Now, at each $x_{i}$, let us pair each cut edge with a non-cut edge. Then it is easy to verify that this pairing results in a connected graph when $\mathcal{O}_{1}$ is applied to $e$.

In a connected graph, a block is a maximal 2-connected subgraph. Let $x$ and $y$ be vertices and let $P$ be path between $x$ and $y$. Suppose edges of $P$ belong to $l$ distinct blocks. Then it is easy to see that $l$ depends only on $x$ and $y$, it is independent of the choice of $P$. In the following, this number $l$ will be denoted by $l(x, y)$.

Proof of Theorem 3.1. We prove the theorem by induction on $|V(G)|$. If $|V(G)|=2$, then the theorem is trivially true. Next, we consider the general case when $|V(G)|>2$. Since each $\mathcal{O}_{i},(i=1,2,3,4)$ reduces the number of vertices by two, and the results of these operations are always 5 -regular and planar, we only need to show that

(a) at least one of $\mathcal{O}_{1}, \mathcal{O}_{3}$, and $\mathcal{O}_{4}$ can be applied to $G$ to result in a connected graph $H$; and

(b) at least one of $\mathcal{O}_{1}, \mathcal{O}_{2}$, and $\mathcal{O}_{4}$ can be applied to $G$ to result in a connected graph $H \neq 5 K_{2}$.

Equivalently, we only need to show that

(i) either $\mathcal{O}_{1}$ or $\mathcal{O}_{4}$ can be applied to $G$ to result in a connected graph $H \neq 5 K_{2}$; or

(ii) both $\mathcal{O}_{2}$ and $\mathcal{O}_{3}$ can be applied to $G$ to result in a connected graph $H$ and $H \neq 5 K_{2}$ if $\mathcal{O}_{2}$ is applied.

Suppose $\mathcal{O}_{1}$ can be applied to an edge of $G$ to produce a connected graph $H$. If $H \neq 5 K_{2}$, then (i) is satisfied. If $H=5 K_{2}$, then $|V(G)|=4$ and Lemma 2.2 implies that either (i) or (ii) is satisfied. Thus we may assume in the following that all outcomes of $\mathcal{O}_{1}$ are disconnected.

Suppose $G \backslash x$ is connected for all $x \in V(G)$. Since $|V(G)|>2, G$ can not have cut edges. Thus we deduce from Lemma 3.2 that $G$ has no simple edges. Therefore, by Lemma 2.1, $G$ can only be an alternating cycle. Now it is clear that (ii) holds.

Suppose $G \backslash x$ is disconnected for at least one vertex $x \in V(G)$. Then among all such vertices, which are called cut vertices, we choose a special 
one as follows. First, let $x_{0}$ be a non-cut vertex. Then let $x$ be a cut vertex such that $l\left(x_{0}, x\right)$ is maximized. Let $C_{0}, C_{1}, \ldots, C_{p}$ be the components of $G \backslash x$, where $C_{0}$ is the component that contains $x_{0}$. For each $i>0$, let $B_{i}$ be the subgraph of $G$ with vertex set $V\left(C_{i}\right) \cup\{x\}$, and edge set $E\left(C_{i}\right) \cup F_{i}$, where $F_{i}$ is the set of edges of $G$ of the form $x y$ with $y \in V\left(C_{i}\right)$. From the choice of $x$ it is clear that $x$ is the only cut vertex of $G$ that is contained in $V\left(B_{i}\right)$. Therefore, if $e \in E\left(B_{i}\right)$ is a cut edge of $G$, one end of $e$ must be $x$ and the other end of $e$ must be incident with two loops. Suppose $e=x y$ is such a cut edge. Since we have assumed that all outcomes of $\mathcal{O}_{1}$ on $e$ are disconnected, we deduce from Lemma 3.1 that all edges of $G$ that are incident with $x$ are cut edges. It follows that $p=4$ and each $B_{i}$, consists of a cut edge and two loops. Clearly, in this case, $\mathcal{O}_{4}$ can be applied to reduce $G$. Notice that the resulting graph $H \neq 5 K_{2}$ since $H$ has a loop. Thus (i) holds in this case. As a consequence, we may assume in the following that no $B_{i}$ contains a cut edge of $G$. This assumption clearly implies that no $B_{i}$ has a singular vertex or a singular edge. Thus we deduce from Lemma 3.2 that no $B_{i}$ has a simple edge. We consider the following two possibilities.

(1) Suppose that every edge in every $B_{i}$ of the form $x y$ is in a parallel family of size two; $x y$ be such an edge of some $B_{i}$. Since $y$ is not a cut vertex of $G$ and $y$ has degree five, $B_{i}$ must have an edge $x z$ with $z \neq y$. Similar to the proof of Lemma 2.1, it is easy to show that $B_{i}$ must consist of $x y, x y, x z, x z$, and an alternating path between $y$ and $z$. Clearly, in this case, both $\mathcal{O}_{2}$ and $\mathcal{O}_{3}$ can be applied to $B_{i}$ and hence to $G$ as well. In addition, it is easy to see that $H \neq 5 K 2$ when $|V(G)| \neq 4$, and $H=K_{2}^{2 L}$ when $|V(G)|=4$. Thus (ii) holds in this case.

(2) Since no $B_{i}$ has simple edges, it remains to consider the case when there is a vertex $y$ in some $B_{i}$ such that there are three or more edges between $x$ and $y$. Since $x$ is a cut vertex while $y$ is not, there must be exactly three edges between $x$ and $y$, and, in addition, there is a loop incident with $y$. Since $G$ has more than two vertices, $x$ must be incident with two other distinct edges $x u, x v$. We prove that $u=v$.

Suppose, on the contrary, that $u \neq v$. Then both $x u$ and $x v$ are simple. It follows that both $u$ and $v$ are in $V\left(C_{0}\right)$, as no $B_{i}$ has simple edges. Consequently, $G$ has cycle $C$ that contains both $x u$ and $x v$, and hence $x u, x v$ are not cut edges. Since $\mathcal{O}_{1}$ can not be applied to $x u$, by Lemma 3.2, $u$ must be a singular vertex. For the same reason, $v$ is also singular. Let $u u_{1}, u u_{2}, u u_{3}$ be the three edges that are incident with $u$ and are not in the cycle $C$. Then, as $u$ is singular, every $e_{i}=u u_{i}$ is a cut edge. For each $i=1,2,3$, let $G_{i}$ be the component of $G \backslash e_{i}$ that contains $u_{i}$. Because of the symmetry between $u$ and $v$, we may assume that $x_{0}$ is not in any $G_{i}$. In addition, since $x$ and 
$u$ are both contained in the cycle $C$, they are contained in the same block. It follows that $l\left(x_{0}, x\right)=l\left(x_{0}, u\right)$. From the choice of $x$ we conclude that no $u_{i}$ can be a cut vertex. Thus each $u_{i}$ is incident with two loops. Therefore, applying $\mathcal{O}_{1}$ to $e_{1}$ can result in a connected graph. This is a contradiction and thus $u=v$ is proved.

Now it is easy to see that (ii) holds in this case, since both $\mathcal{O}_{2}$ and $\mathcal{O}_{3}$ can be applied to $x y$, and the resulting connected graph is not $5 K_{2}$ as it has loops.

\section{2-edge-connected 5-regular planar graphs}

Both of the following two graph operations will be needed to reduce graphs in $\mathcal{P}_{2}$.

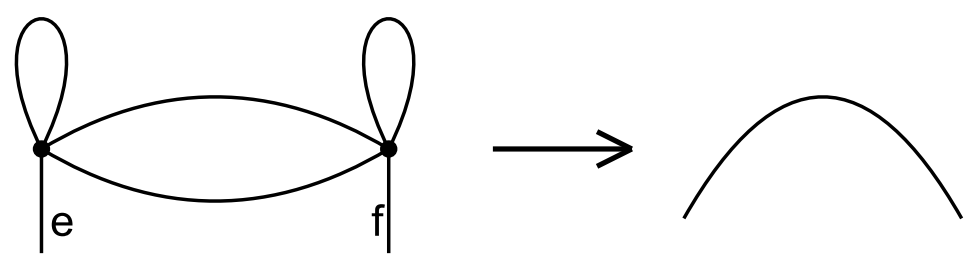

Figure 4.1: Operation $\mathcal{O}_{5}$.

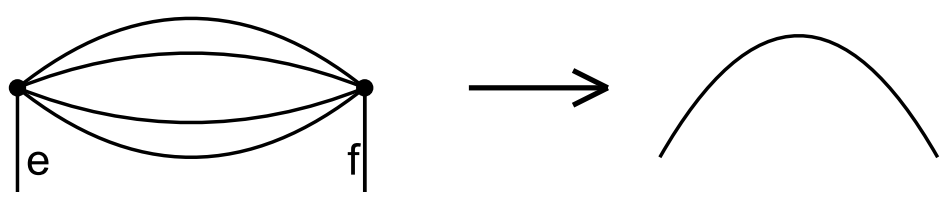

Figure 4.2: Operation $\mathcal{O}_{6}$.

Here, $e$ and $f$ are distinct simple edges. We also remark that the 2-edgeconnectivity is preserved under these operations.

Theorem 4.1 Every graph in $\mathcal{P}_{2}$ can be reduced within $\mathcal{P}_{2}$ by $\mathcal{O}_{1}, \mathcal{O}_{2}, \mathcal{O}_{3}$, $\mathcal{O}_{5}$, and $\mathcal{O}_{6}$ to $3 K_{2}^{L}$ or $5 K_{2}$. 
We point out that operations $\mathcal{O}_{2}$ and $\mathcal{O}_{3}$ are only used in very special ways. In fact, they are only used to shorten an alternating path in a graph. To prove Theorem 4.1, we need to prove a few lemmas. For any two disjoint vertex sets $X$ and $Y$ of a graph $G$, let $m_{G}(X, Y)$, or simply $m(X, Y)$ if there is no ambiguity, be the number of edges with one end in $X$ and one end in $Y$. In case $Y=V(G)-X$, we may also denote $m(X, Y)$ by $m(X)$ or by $m(Y)$.

It is well known that the cut function of a graph is submodular (Corollary 3.1a [14]). This property is used very widely to uncross (simplify) a given collection of cuts. The following is a refinement of this property in a special situation.

Lemma 4.1 Let $X_{1}, X_{2}, X_{3}, X_{4}$ be a partition of $V(G)$ such that $X_{i} \neq \emptyset$ for all $i$. Let $p$ be an integer for which $m\left(X_{1} \cup X_{2}\right) \leq p, m\left(X_{1} \cup X_{4}\right) \leq p$, and $m\left(X_{i}\right) \geq p$ for all $i$. Then $p$ is even, $m\left(X_{1}, X_{3}\right)=m\left(X_{2}, X_{4}\right)=0$, and $m\left(X_{1}, X_{2}\right)=m\left(X_{2}, X_{3}\right)=m\left(X_{3}, X_{4}\right)=m\left(X_{4}, X_{1}\right)=p / 2$.

Proof. For $i<j$, let $m_{i j}=m\left(X_{i}, X_{j}\right)$. Then we have

$2 p \geq m\left(X_{1} \cup X_{2}\right)+m\left(X_{1} \cup X_{4}\right)=m_{12}+m_{23}+m_{34}+m_{14}+2 m_{13}+2 m_{24}$.

Consequently,

$$
2 p \geq\left(m_{12}+m_{13}+m_{14}\right)+\left(m_{13}+m_{23}+m_{34}\right)=m\left(X_{1}\right)+m\left(X_{3}\right) \geq 2 p,
$$

and thus we conclude that

$$
m\left(X_{1} \cup X_{2}\right)=m\left(X_{1} \cup X_{4}\right)=m\left(X_{1}\right)=m\left(X_{3}\right)=p \text { and } m_{24}=0 .
$$

Similarly, we also have

$$
2 p \geq\left(m_{12}+m_{23}+m_{24}\right)+\left(m_{14}+m_{24}+m_{34}\right)=m\left(X_{2}\right)+m\left(X_{4}\right) \geq 2 p,
$$

and thus

$$
m\left(X_{2}\right)=m\left(X_{4}\right)=p \text { and } m_{13}=0 .
$$

Now, notice that the equations

$$
m\left(X_{1} \cup X_{2}\right)=m\left(X_{1}\right)=m\left(X_{2}\right)=m\left(X_{3}\right)=p
$$

which can be rewritten as

$$
m_{14}+m_{23}=m_{12}+m_{14}=m_{12}+m_{23}=m_{23}+m_{34}=p
$$

have a unique solution 


$$
m_{12}=m_{23}=m_{34}=m_{14}=p / 2 .
$$

Thus $p$ must be even and the lemma is proved.

Next, we consider a generalization of Lemma 3.1 to higher edge-connectivity. Suppose a vertex $x$ of a plane graph $G$ is incident with four simple edges $x x_{1}, x x_{2}, x x_{3}$, and $x x_{4}$ where the edges are listed in the same cyclic order as drawn on the plane. Then a quadrilateral partition of $G$, with center $x$ and width $w$, is a partition $X_{1}, X_{2}, X_{3}, X_{4}$ of $V(G \backslash x)$ such that $x_{i} \in X_{i}$ for all $i, m_{G \backslash x}\left(X_{1}, X_{2}\right)=m_{G \backslash x}\left(X_{2}, X_{3}\right)=m_{G \backslash x}\left(X_{3}, X_{4}\right)=m_{G \backslash x}\left(X_{4}, X_{1}\right)=w$, and $m_{G} \backslash x\left(X_{1}, X_{3}\right)=m_{G} \backslash x\left(X_{2}, X_{4}\right)=0$. Notice that the conclusion of Lemma 3.1 can be restated as; if both splittings at $\mathrm{x}$ are disconnected, then $G$ has a quadrilateral partition, with center $x$ and width 0 . Our next result generalizes Lemma 3.1. For a set $X$ of vertices of a graph $G$, let $E_{G}(X)$ denote the set of edges of $G$ with one end in $X$ and one end in $V(G)-X$.

Lemma 4.2 Let $t$ be a positive integer and let $G$ be a connected plane graph with $|V(G)| \geq 2$. Let $x$ be a degree-four vertex of $G$ such that $\left|E_{G}(X)\right| \geq t$ for all $X \subseteq V(G)$ with $X \neq \emptyset,\{x\}, V(G)-\{x\}$, or $V(G)$. If both splits of $G$ at $x$ are not $t$-edge-connected, then $t$ is odd, and $G$ has a quadrilateral partition with center $x$ and width $(t-1) / 2$.

Proof. We first prove that no loop is incident with $x$. Suppose, on the contrary, that $x$ is incident with a loop $e$. Then there is only one outcome when we split $x$. This outcome, say $H$, is obtained from $G$ by deleting $e$ and then contracting another edge incident with $x$. Observe that $E_{H}(Z)=E_{G}(Z)$ whenever $Z$ is a non-empty set of vertices of $H$ such that the new vertex is not in $Z$. It follows that $H$ is $t$-edge-connected, a contradiction. In the following, we assume that no edge incident with $x$ is a loop.

Let $x x_{1}, x x_{2}, x x_{3}, x x_{4}$ be the four edges incident with $x$, where the edges are listed in the same cyclic order as drawn on the plane. Let $X=\left\{x_{1}, x_{2}, x_{3}\right.$, $\left.x_{4}\right\}$. We first consider the split $\left\{x x_{1}, x x_{2}\right\}-\left\{x x_{3}, x x_{4}\right\}$. Since the result $G_{1}$ of this split is not $t$-edge-connected, $V\left(G_{1}\right)=V(G \backslash x)$ has a partition $\left(V_{1}, V_{2}\right)$ such that $V_{i} \neq \emptyset(i=1,2)$ and $E_{G_{1}}\left(V_{1}\right)=E_{G_{1}}\left(V_{2}\right)$ has at most $t-1$ edges. If some $V_{i}$, say $V_{1}$, contains vertices from both $\left\{x_{1}, x_{2}\right\}$ and $\left\{x_{3}, x_{4}\right\}$, then it is easy to see that $E_{G}\left(V_{2}\right)=E_{G_{1}}\left(V_{2}\right)$, which implies $t \leq\left|E_{G}\left(V_{2}\right)\right|=\left|E_{G_{1}}\left(V_{2}\right)\right| \leq t-1$, a contradiction. Therefore, each $V_{i}$ contains only vertices from one of $\left\{x_{1}, x_{2}\right\}$ and $\left\{x_{3}, x_{4}\right\}$. Without loss of generality, let us assume that $\left\{x_{1}, x_{2}\right\} \subseteq V_{1}$ and $\left\{x_{3}, x_{4}\right\} \subseteq V_{2}$. Suppose the split $\left\{x x_{1}, x x_{4}\right\}-\left\{x x_{2}, x x_{3}\right\}$ results in a graph $G_{2}$. Since $G_{2}$ 
is not $t$-edge-connected, $V\left(G_{2}\right)=V\left(G_{1}\right)$ has a partition $\left(U_{1}, U_{2}\right)$ such that $U_{i} \neq \emptyset$ and $\left|E_{G_{2}}\left(U_{i}\right)\right| \leq t-1$ for $i=1,2$. With the same argument, we may assume that $\left\{x_{1}, x_{4}\right\} \subseteq U_{1}$ and $\left\{x_{2}, x_{3}\right\} \subseteq U_{2}$. Let $X_{1}=V_{1} \cap U_{1}, X_{2}=V_{1} \cap U_{2}, X_{3}=V_{2} \cap U_{2}$, and $X_{4}=V_{2} \cap U_{1}$. Then $\left(X_{1}, X_{2}, X_{3}, X_{4}\right)$ is a partition of $V(G \backslash x)$ and $x_{i} \in X_{i}$ for $i=1,2,3$, 4. Observe that, for each $i$, we have $\left|E_{G \backslash x}\left(X_{i}\right)\right|=\left|E_{G}\left(X_{i}\right)\right|-1 \geq t-1$. Thus we may apply Lemma 4.1 to $G \backslash x$, with $p=t-1$. It follows that $t-1$ is even, which means $t$ is odd, and $\left(X_{1}, X_{2}, X_{3}, X_{4}\right)$ is a quadrilateral partition with center $x$ and width $(t-1) / 2$.

This lemma will be used several times. In this section, it is used via the following lemma.

Lemma 4.3 If $e$ is a simple edge of a graph $G$ in $\mathcal{P}_{t}(t=2,4)$ such that $G \backslash e$ is t-edge-connected, then at least one outcome of $\mathcal{O}_{1}$ on $e$ is still t-edgeconnected.

Proof. Let $e=x y$. By Lemma $4.2, x$ can be split in $G \backslash e$ to result in a $t$-edge-connected graph $H$. By Lemma 4.2 again, $y$ can be split in $H$ to result a $t$-edge-connected graph $H^{\prime}$. Clearly, $H^{\prime}$ is an outcome of $\mathcal{O}_{1}$ on $e$ and thus Lemma 4.3 is proved.

A set $F$ of edges of a connected graph $G$ is a $t$-edge-cut if $|F|=t$ and $G \backslash F$ is disconnected. When $G$ is $t$-edge-connected, it is easy to see that, for each $t$-edge-cut $F$ of $G$, the graph $G \backslash F$ has exactly two components. Suppose $F$ is a $t$-edge-cut such that smaller (in terms of the number of vertices) component $G_{1}$ of $G \backslash F$ is the smallest among all $t$-edge-cuts of $G$. Then we call $G_{1}$ an end of $G$ and $F$ a critical $t$-edge-cut.

Lemma 4.4 Let $H$ be an end of a t-edge-connected graph $G$. Then no edge of $H$ is contained in a $t$-edge-cut of $G$.

Proof. Suppose, on the contrary, that some edge $e$ of $H$ is contained in a $t$-edge-cut $F$ of $G$. Let $U=V(H)$ and let $U^{\prime}=V\left(H^{\prime}\right)$ for some component $H^{\prime}$ of $G \backslash F$. Let $X_{1}=U \cap U^{\prime}, X_{2}=U-U^{\prime}, X_{3}=V(G)-U-U^{\prime}$, and $X 4=U^{\prime}-U$. Then $X_{1} \neq \emptyset \neq X_{2}$ as $e \in E_{G}\left(X_{1}, X_{2}\right)$. If $X_{3} \neq \emptyset$ or $X_{4} \neq \emptyset$, then one component of $G \backslash F$ is a proper subgraph of $H$, contradicting the minimality of $H$. Thus we may assume $X_{3} \neq \emptyset \neq X_{4}$. Notice that Lemma 4.2 can be applied to $\left(X_{1}, X_{2}, X_{3}, X_{4}\right)$, with $p=t$. From this lemma we conclude that $E_{G}\left(X_{1}\right)$ is a $t$-edge-cut of $G$ and one component of $G \backslash E_{G}\left(X_{1}\right)$ 
is a proper subgraph of $H$. Again, it contradicts the minimality of $H$ and thus Lemma 4.4 is proved.

Proof of Theorem 4.1 We prove the theorem by induction on $|V(G)|$. If $|V(G)|=2$, then $G=3 K_{2}^{L}$ or $5 K_{2}$ and thus the theorem is trivially true. Next, we consider the general case when $|V(G)|>2$. Since each $\mathcal{O}_{i}(i=1,2,3,5,6)$ reduces the number of vertices by two, and the results of these operations are always 5 -regular and planar, we only need to show that

$\left(^{*}\right) G$ can be reduced to a 2-edge-connected graph by applying $\mathcal{O}_{1}, \mathcal{O}_{2}$, $\mathcal{O}_{3}, \mathcal{O}_{5}$, or $\mathcal{O}_{6}$ once.

If $G$ has no simple edges, then (*) follows from Lemma 2.1. Thus we may assume that $G$ has at least one simple edge $e$. If $G$ has a simple edge $e$ such that $G \backslash e$ is 2 -edge-connected, then $\left(^{*}\right)$ follows from Lemma 4.3. Thus we may assume that every simple edge is contained in a 2-edge-cut. In particular, $G$ has a critical 2-edge-cut, say $\{e, f\}$. Let $H$ be the corresponding end. Then we conclude from Lemma 4.4 that $H$ has no simple edges. Let us assume $e=x x^{\prime}$ and $f=y y^{\prime}$, where $x, y \in V(H)$. If $H$ does not have any other vertices, then $\left(^{*}\right)$ holds since it is easy to see that $x \neq y$, and either $\mathcal{O}_{5}$ or $\mathcal{O}_{6}$ can be applied to $G$. Thus we may assume that $H$ has a vertex $z \neq x, y$. Since all edges incident with $z$ are not simple, $z$ can be adjacent to at most two vertices. If $z$ is adjacent to only one vertex $z^{\prime}$, then there are three edges from $z$ to $z^{\prime}$, and there is a loop incident with $z$. Clearly, in this case, if $\mathcal{O}_{2}$ is applied to $z z^{\prime}$, then the resulting graph is 2-edge-connected and thus $\left(^{*}\right)$ is proved. Therefore, we may assume that $z$ is adjacent to two vertices $z^{\prime}$ and $z^{\prime \prime}$, Observe that, in this case, one of $z z^{\prime}$ and $z z^{\prime \prime}$ is contained in a parallel family of size two and the other is in a parallel family of size three. It follows that $\mathcal{O}_{3}$ can be applied in.this case and thus $\left(^{*}\right)$ is proved again.

\section{3-edge-connected 5-regular planar graphs}

First we observe that, if $G$ is a graph in $\mathcal{P}_{3}$, then each parallel family of $G$ has at most three edges (unless $G=5 K_{2}$ ), and $G \backslash x$ is connected for all vertices $x$ of $G$. In this section, two more graph operations are needed.

Here, $e, f$ and $g$ are distinct simple edges. We remark that, since they are results of contracting edges, they both preserve edge-connectivity.

Theorem 5.1 Every graph in $\mathcal{P}_{3}$ can be reduced within $\mathcal{P}_{3}$ by $\mathcal{O}_{1}, \mathcal{O}_{3}, \mathcal{O}_{7}$, and $\mathcal{O}_{8}$ to $5 K_{2}$ or $3 K_{2}^{L}$. 


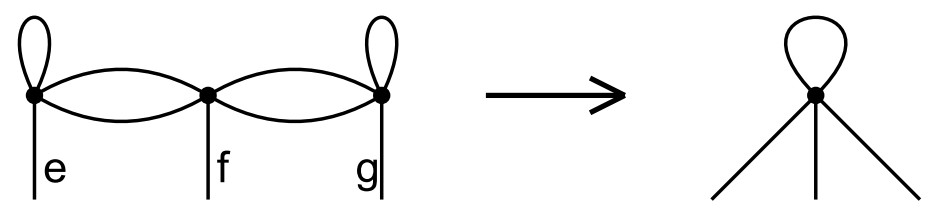

Figure 5.1: Operation $\mathcal{O}_{7}$.
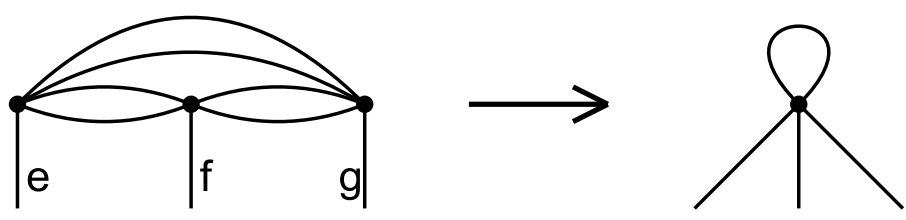

Figure 5.2: Operation $\mathcal{O}_{8}$

To prove Theorem 5.1, we need to prove some lemmas. The first is an analog of Lemma 4.1, which is a specification of Lemma 4.2 for $t=3$.

Lemma 5.1 Let $x$ be a degree-four vertex of 3-edge-connected plane graph $G$ with $|V(G)| \geq 2$. If no split of $G$ at $x$ is 3-edge-connected, then $G$ has a quadrilateral partition with center $x$ and width 1.

The next lemma is an analog of Lemma 3.2.

Lemma 5.2 Let $e=x y$ be a simple edge of $G \in \mathcal{P}_{3}$ such that $G \backslash e$ is also 3 -edge-connected. Suppose no quadrilateral partitions of $G \backslash e$ centered at $x$ or $y$ has width 1 . If no outcome of $\mathcal{O}_{1}$ on e is 3-edge-connected, then $V(G)-$ $\{x, y\}$ has a partition $X_{1}, X_{2}, X_{3}, X_{4}$ such that $m\left(X_{1}, X_{2}\right)=m\left(X_{3}, X_{4}\right)=1$, $m\left(\{y\}, X_{i}\right)=1$ for all $i$, and at least one of the following holds.

(a) $m\left(\{x\}, X_{i}\right)=1$ for all $i$;

(b) $m\left(X_{2}, X_{3}\right)=1, m\left(\{x\}, X_{1}\right)=m\left(\{x\}, X_{4}\right)=1$, and $m\left(\{x\}, X_{3}\right)=2$;

(c) $m\left(X_{2}, X_{3}\right)=m\left(X_{1}, X_{4}\right)=1$ and $m\left(\{x\}, X_{1}\right)=m\left(\{x\}, X_{4}\right)=2$. 
Remark. If we allow $G$ to be non-planar, then there is a fourth alternative:

(d) $m\left(X_{2}, X_{3}\right)=m\left(X_{1}, X_{4}\right)=1$ and $m\left(\{x\}, X_{1}\right)=m\left(\{x\}, X_{3}\right)=2$.

Proof of Lemma 5.2. By Lemma 5.1, $G \backslash e$ can be split at $x$ to result in a 3-edge-connected graph $H$. Let $f_{1}$ and $f_{2}$ be the two new edges. Since no outcome of $\mathcal{O}_{1}$ on $e$ is 3-edge-connected, no split of $H$ at $y$ is 3edge-connected. By Lemma 5.1, $H$ has a quadrilateral partition with center $y$ and width 1 . If some $f_{i}$, say $f_{1}$, is between some $X_{i}$ and $X_{j}$, say $X_{1}$ and $X_{4}$, Then $f_{2}$ is not incident with any vertex in $X_{1} \cup X_{4}$, as no quadrilateral partition of $G \backslash e$ centered at $y$ has width 1 . It follows that either $f_{2} \in E_{H}\left(X_{2}, X_{3}\right)$, which implies (a), or both ends of $f_{2}$ are in $X_{i}(i=2,3)$, which implies (b). Therefore, we may assume that, for each $f_{i}$, its two ends are both contained in some $X_{j}$. Notice that $f_{1}$ and $f_{2}$ can not be contained in the same $X_{j}$, as no quadrilateral partition of $G \backslash e$ centered at $y$ has width 1. Now it is clear that there are two possibilities, one yields (c) and one yields (d).

Let $\left(X_{1}, X_{2}\right)$ be a partition of the vertex set of a graph $G$ and let

$$
E_{G}\left(X_{1}\right)=\left\{x_{1 j} x_{2 j}: x_{1 j} \in X_{1}, x_{2 j} \in X_{2}, j=1,2, \ldots, t\right\}
$$

Then, for $i=1,2$, we define $G_{i}$ to be the graph obtained from $G \backslash X_{k}$, where $k \neq i$, by adding a new vertex $x_{i}$ and $t$ new edges $x_{i} x_{i j}$ for all $j$.

Lemma 5.3 $G$ is t-edge-connected if and only if both $G_{1}$ and $G_{2}$ are $t$-edgeconnected.

Proof. Suppose $G$ is $t$-edge-connected. Then both $G \backslash X_{1}$ and $G \backslash X_{2}$ are connected. Thus each $G_{i}$ can be considered as obtained from $G$ by contracting edges of $G \backslash X_{i}$. Since contracting edges does not change edgeconnectivity, it follows that each $G_{i}$ is $t$-edge-connected. Next, we assume that both $G_{1}$ and $G_{2}$ are $t$-edge-connected. We need to show that $\mid E_{G}(Y) \geq t$ for all $Y \neq \emptyset, V(G)$. Suppose, on the contrary, that $\left|E_{G}(Y)\right|<t$ for some $Y \neq \emptyset, V(G)$. Let $Z_{1}=X_{1} \cap Y, Z_{2}=X_{2} \cap Y, Z_{3}=X_{2}-Y$, and $Z_{4}=X_{1}-Y$. Since both $G_{1}$ and $G_{2}$ are $t$-edge-connected, no $Z_{i}$ can be empty. Notice that each $Z_{i}$ is a subset of some $X_{k}$, it follows that $E_{G}\left(Z_{i}\right)=E_{G_{k}}\left(Z_{i}\right)$ and thus $\mid E_{G}\left(Z_{i}\right) \geq t$. Let $F$ be the set of all edges between different $Z_{i}$ and $Z_{j}$. Then the sum of $m_{G}\left(Z_{i}\right)$, over all $i$, is $2|F|$, and it follows that $|F| \geq 2 t$. On the other hand, $|F| \leq\left|E_{G}\left(X_{1}\right)\right|+\left|E_{G}(Y)\right|<2 t$, 
a contradiction which completes the proof.

Let $G \in \mathcal{P}_{3}$. A 3-edge-cut $F$ of $G$ is trivial if there is a vertex incident with all three edges in $F$. Observe that this vertex must also be incident with a loop.

Lemma 5.4 Let $G \in \mathcal{P}_{3}$ with $|V(G)| \geq 4$. Suppose $G$ has a loop at $z \in$ $V(G)$ and suppose all 3-edge-cuts of $G$ are trivial. Then at least one of the following holds.

(a) $G$ has an edge e, which is not incident with $z$, such that an outcome of $\mathcal{O}_{1}$ on e is in $\mathcal{P}_{3}$;

(b) there are three distinct vertices $u, v, w$ in $V(G \backslash z)$ such that the five edges incident with $v$ are $u v, u v, v w, v w, v w$;

(c) $V(G)=\{z, u, v, w\}$, and, for $E^{\prime}=\{z z, z u, z v, z w, u v, u v, v w, v w\}$, we have either $E(G)=\{u u, w w\} \cup E^{\prime}$ or $E(G)=\{u w, u w\} \cup E^{\prime}$.

Proof. Suppose $G \backslash z$ has no simple edges. If all vertices of $G \backslash z$ are adjacent to $z$, graph $G$ must have exactly four vertices, say $z, u, v, w$. Since $G$ is 3-edge-connected, at least two pairs of distinct vertices in $\{u, v, w\}$ are adjacent. Then it is easy to verify that (c) holds in this case. Thus we may assume that $G$ has a vertex $v$ not adjacent to $z$. Notice that no loop is incident with $v$, because otherwise, the only vertex adjacent to $v$ would be a cut vertex, which is a contradiction. It follows that $v$ is adjacent to exactly two vertices and thus (b) holds. Next, we assume that $G \backslash z$ has at least one simple edge. We will consider three cases.

Suppose the simple edges of $G \backslash z$ are not incident with any loops. Then we consider an arbitrary simple edge e of $G \backslash z$, which does exist by the above assumption. Observed that $e$ is not contained in any 3-edge-cuts, as all such cuts are trivial. It follows that $G \backslash e$ must be 3-edge-connected. Then we deduce from Lemma 5.2 that at least one outcome of $\mathcal{O}_{1}$ on $e$ is 3 -edge-connected. Thus (a) holds in this case.

Suppose $G \backslash z$ has a simple edge $e=x y$ such that both $x$ and $y$ are incident with loops. Then the only 3-edge-cuts of $G$ that contain $e$ are $E_{G}(\{x\})$ and $E_{G}(\{y\})$. It follows that the only 2-edge-cuts of $G \backslash e$ are $E_{G \backslash e}(\{x\})$ and $E_{G \backslash e}(\{y\})$. Notice that the only outcome of $\mathcal{O}_{1}$ on $e$, say $H$, can be obtained from $G \backslash e$ by deleting loops $x x$, yy and then contracting two edges, one from $E_{G \backslash e}(\{x\})$ and one from $E_{G \backslash e}(\{y\})$. Thus $H$ is 3-edge-connected and (a) holds in this case. 
Suppose the last two cases do not happen. Then every simple edge of $G \backslash z$ is incident with at most one loop. In addition, $G \backslash z$ does have an edge $e=x y$, for which $x$ is incident with a loop while $y$ is not. Let us choose this $e$ so that both $x$ and $y$ are adjacent to $z$, if it is possible. Then we prove that at least one outcome of $\mathcal{O}_{1}$ on $e$ is 3-edge-connected. First observe that the only 3-edge-cut of $G$ that contains $e$ is $E_{G}(\{x\})$. It follows that the only 2-edge-cut of $G \backslash e$ is $E_{G \backslash e}(\{x\})$. Therefore, the graph obtained from $G \backslash e$ by deleting the loop $x x$ and then contracting an edge incident with $x$ is 3 -edge-connected. Let $H$ be this graph and let $f$ be the new edge. We need to show that at least one split of $H$ at $y$ is 3-edge-connected. Suppose not. Then, by Lemma 5.1, $H$ has a quadrilateral partition $\left(X_{1}, X_{2}, X_{3}, X_{4}\right)$, with center $y$ and width 1 . Let us assume that $f$ is incident with a vertex in $X_{4}$. Then it is easy to see that $\left|X_{i}\right|=1$ for $i=1,2,3$. It follows from the choice of $e$ that $X_{2} \neq\{z\}$. By symmetry, we may also assume that $X_{1} \neq\{z\}$. Therefore, the only edge between $X_{1}$ and $X_{2}$ is simple and is incident with two loops, a contradiction.

Proof of Theorem 5.1. We prove the theorem by induction on $|V(G)|$. If $|V(G)|=2$, then $G=3 K_{2}^{L}$ or $5 K_{2}$ and thus the theorem is trivially true. Next, we consider the general case when $|V(G)|>2$. Since each $\mathcal{O}_{i}(i=1,3,7,8)$ reduces the number of vertices by two, and the results of these operations are always 5 -regular and planar, we only need to show that

(*) $G$ can be reduced to a 3 -edge-connected graph by applying $\mathcal{O}_{1}, \mathcal{O}_{3}, \mathcal{O}_{7}$, or $\mathcal{O}_{8}$ once.

Suppose $G$ is 4-edge-connected. If $G$ has no simple edges, then Lemma 2.1 implies that $G$ is an alternating cycle. Clearly, $\mathcal{O}_{3}$ can be applied in the case and thus $\left(^{*}\right)$ holds. If $G$ does have a simple edge $e$, then we deduce from Lemma 5.2 that at least one outcome of $\mathcal{O}_{1}$ on $e$ is 3-edge-connected and thus $\left(^{*}\right)$ holds again. In the following, Let us assume that $G$ has at least one 3-edge-cut.

Suppose all 3-edge-cuts of $G$ are trivial. Then, under the above assumption, $G$ has a loop at a vertex $z$, Let us apply Lemma 5.4 to $G$. Then it is easy to see that $(*)$ holds because, if (a) occurs then $\mathcal{O}_{1}$ can be applied; if (b) occurs then $\mathcal{O}_{3}$ can be applied; and if (c) occurs then $\mathcal{O}_{7}$ or $\mathcal{O}_{8}$ can be applied. Thus we may assume further that $G$ has a nontrivial 3-edge-cut.

Choose a nontrivial 3-edge-cut $F=\left\{e_{1}, e_{2}, e_{3}\right\}$ such that the smaller component $J$ of $G \backslash F$ is as small as possible. Let us define $G_{1}$ and $G_{2}$ as in Lemma 5.3 such that $G_{1} \backslash x_{1}=J$. Let $H$ be obtained from $G_{1}$ by adding a loop $e_{0}$ to $x_{1}$. Then we deduce from Lemma 5.3 that $H \in \mathcal{P}_{3}$. Notice that 
every nontrivial 3-edge-cut of $H$ is also a nontrivial 3-edge-cut of $G$. Thus the minimality of $J$ implies that all 3-edge-cuts of $H$ are trivial. Now let us apply Lemma 5.4 to $H$, with $z=x_{i}$. If (b) or (c) occurs, then $\mathcal{O}_{3}, \mathcal{O}_{7}$, or $\mathcal{O}_{8}$ can be applied to $H$ and so can also be applied to $G$. Since these three operations preserve edge-connectivity, $\left(^{*}\right)$ holds in this case. If (a) occurs, then $\mathcal{O}_{1}$ can be applied to $H$, on an edge e which is not incident with $x_{1}$, such that the resulting graph $H^{\prime}$ is in $\mathcal{P}_{3}$. Since $x_{1}$ is still a vertex of $H^{\prime}$ and $H^{\prime}$ is 3-edge-connected, $e_{1}, e_{2}, e_{3}$ must still be edges of $H^{\prime}$. Let $G^{\prime}$ be obtained from $G_{2}$ and $H^{\prime} \backslash e_{0}$ by identifying $e_{1}, e_{2}, e_{3}$, in the same way as in Lemma 5.3, then the lemma implies that $G^{\prime}$ is 3-edge-connected. In fact, it is not difficult to see that $G^{\prime}$ is the exactly the resulting graph if we apply $\mathcal{O}_{1}$ to $G$, on the edge $e$. Therefore $\left(^{*}\right)$ holds again in this case and thus the proof of Theorem 5.1 is complete.

\section{4-edge-connected 5-regular planar graphs}

Let $G \in \mathcal{P}_{4}$. Since $G$ is also a graph in $\mathcal{P}_{3}$, we know that $G$ is 2-connected and each parallel family of $G$ has size at most three (unless $G=5 K_{2}$ ). In addition, it is easy to see that $G$ has no loops. Next, we define two new operations.
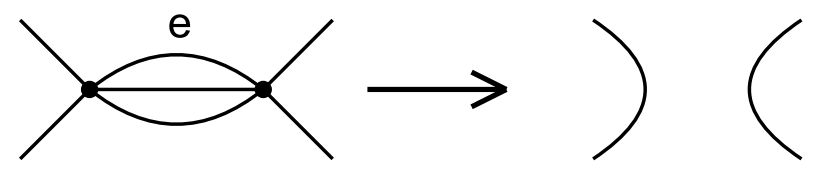

or

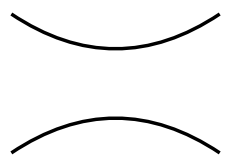

Figure 6.1: Operation $\mathcal{O}_{9}$.

Here, $e$ is in a parallel family of size three. This operation clearly includes $\mathcal{O}_{2}$. This is the only operation other than $\mathcal{O}_{1}$ that depends on the drawing of the graph. We need to point out that $\mathcal{O}_{2}$ is actually the removal of a triple hand in one way, while $\mathcal{O}_{9}$ is offering both possibilities.

Operation $\mathcal{O}_{10}$ is quite clear from its definition. Unlike all previous operations, this operation reduces the number of edges by ten. In general, $\mathcal{O}_{10}$ may not preserve the edge-connectivity. However, if this operation can 


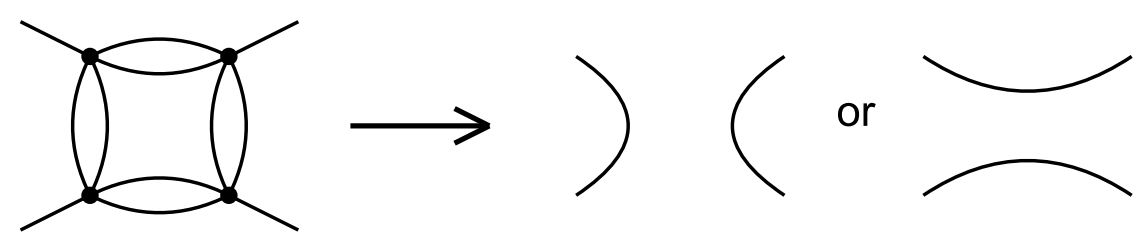

Figure 6.2: Operation $\mathcal{O}_{10}$.

be applied to a graph $G$ in $\mathcal{P}_{4}$ at all, then the result can be considered as obtained from $G$ by contracting the eight edges in the 4-cycle and then splitting the new vertex. Therefore, we deduce from Lemma 4.2 that at least one of the two outcomes of this operation is still 4-edge-connected. Clearly, the same reasoning also applies to $\mathcal{O}_{9}$, These observations are summarized in the following lemma.

Lemma 6.1 For $i=9,10$, if $\mathcal{O}_{i}$ can be applied to a graph in $\mathcal{P}_{4}$, then it can also be applied in such a way that the 4-edge-connectivity is maintained.

The next is the main theorem of this section.

Theorem 6.1 Every graph in $\mathcal{P}_{4}$ can be reduced within $\mathcal{P}_{4}$ by $\mathcal{O}_{1}, \mathcal{O}_{9}$, and $\mathcal{O}_{10}$ to $5 K_{2}$.

Proof. This proof is very similar to that of Theorem 4.1. We prove by induction on $|V(G)|$. If $|V(G)|=2$, then $G=5 K_{2}$ and thus the theorem is trivially true. Next, we consider the general case when $|V(G)|>2$. Since the result of applying each $\mathcal{O}_{i}(i=1,9,10)$ is always 5 -regular and planar, we only need to show that

(*) $G$ can be reduced to a 4-edge-connected graph by applying $\mathcal{O}_{1}, \mathcal{O}_{9}$, or $\mathcal{O}_{10}$ once.

If $G$ has no simple edges, then (*) follows from Lemma 2.1 and Lemma 6.1 since $\mathcal{O}_{9}$ can be applied to $G$. Thus we may assume that $G$ has at least one simple edge $e$. If $G$ has a simple edge $e$ such that $G \backslash e$ is 4-edge-connected, then $\left(^{*}\right)$ follows from Lemma 4.3. Thus we may assume that every simple edge is contained in a 4-edge-cut. In particular, $G$ has a critical 4-edge-cut $F$. Let $H$ be the corresponding end. Then we conclude from Lemma 5.3 
that $H$ has no simple edges. We also conclude from Lemma 6.1 that $H$ has no parallel family of size three. Consequently, each edge of $H$ is contained in a parallel family of size two and thus each vertex of $H$ has an even degree in $H$. Since $G$ is 5-regular, it follows that each vertex of $H$ must be incident with an edge in $F$. Therefore, $H$ can have at most four vertices. Now, it is straightforward to verify that $H$ must be a double-cycle of length four. Notice that $\mathcal{O}_{10}$ can be applied in this case and thus, by Lemma $6.1,\left(^{*}\right)$ is proved again.

\section{5-edge-connected 5-regular planar graphs}

Let $G \in \mathcal{P}_{5}$. Then $G$ has no loops and each parallel family has at most two edges (unless $G=5 K_{2}$ ). In addition, $G$ is 3-connected. The following are the last two operations we need.

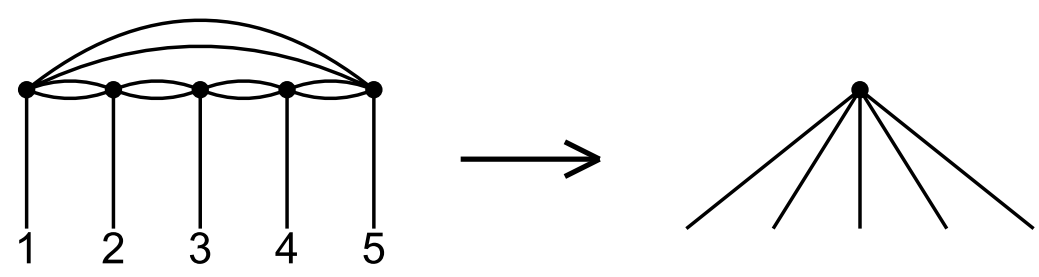

Figure 7.1: Operation $\mathcal{O}_{11}$
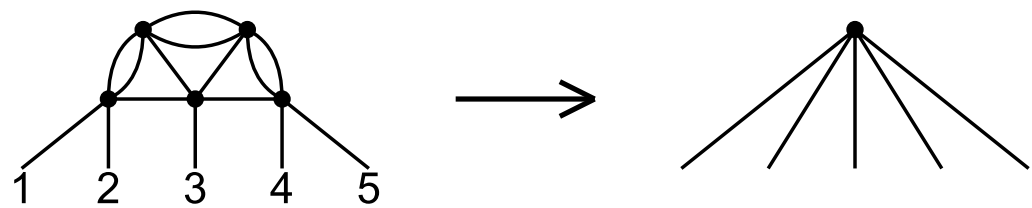

Figure 7.2: Operation $\mathcal{O}_{12}$.

Notice that both operations reduce the number of vertices by four, and both operations preserve edge-connectivity. 
Theorem 7.1 Every graph in $\mathcal{P}_{5}$ can be reduced within $\mathcal{P}_{5}$ by $\mathcal{O}_{1}, \mathcal{O}_{11}$, and $\mathcal{O}_{12}$ to $5 K_{2}$.

To prove the theorem, we need a lemma similar to Lemma 4.2.

Lemma 7.1 Let $t$ be a positive integer and let $G$ be a connected plane graph with $|V(G)| \geq 2$. Let $x, y$ be two degree-four vertices of $G$ such that $x y$ is not an edge of $G$ and $\left|E_{G}(X)\right| \geq t$ for all $X \subseteq V(G)$ with $X \neq \emptyset,\{x\},\{y\}, V(G)-\{x\}, V(G)-\{y\}$, or $V(G)$. Let $H_{1}, H_{2}$ be the two outcomes after splitting $x$. If there are $X_{1}, X_{2}$ such that for each $i=1,2$, $\left|E_{H_{i}}\left(X_{i}\right)\right|<t$ where $X_{i} \subseteq V\left(H_{i}\right)$ with $X_{i} \neq \emptyset,\{y\}, V\left(H_{i}\right)-\{y\}$, or $V\left(H_{i}\right)$, then $t$ is odd, and $G$ has a quadrilateral partition with center $x$ and width $(t-1) / 2$.

We omit the proof of Lemma 7.1 since the proof is similar to Lemma 4.2.

A 5-edge-cut is trivial if it is incident with a single vertex. There is a graph in $\mathcal{P}_{5}$ with 6 vertices, which we call $W_{5}^{\prime}$, illustrated in Figure 7.3.

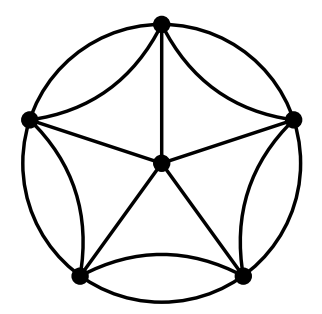

Figure 7.3: $W_{5}^{\prime}$.

Lemma 7.2 Let $G \in \mathcal{P}_{5}, G \neq 5 K_{2}$, and $z \in V(G)$. Suppose every 5-edgecut in $G$ is trivial. If $\mathcal{O}_{1}$ cannot be applied within $\mathcal{P}_{5}$ to any simple edge that is not incident with $z$, then $G=W_{5}^{\prime}$.

Proof. Every vertex in $G$ is incident with at least one simple edge. If every simple edge is incident with $z$, then $|V(G)|=6$ and $G=W_{5}^{\prime}$.

Otherwise, there is a simple edge $e=x y$ that is not incident with $z$. We claim that $\left|E_{G \backslash e}(X)\right| \geq 5$ for all $X \subseteq V(G \backslash e)=V(G)$ with $X \neq$ $\emptyset,\{x\},\{y\}, V(G)-\{x\}, V(G)-\{y\}$, or $V(G)$. If there is a vertex set $X^{\prime} \subseteq$ $V(G)$ with $X^{\prime} \neq \emptyset, V(G)$ such that $\left|E_{G \backslash e}\left(X^{\prime}\right)\right|<5$ holds, then $\left|E_{G}\left(X^{\prime}\right)\right| \leq$ 
$\left|E_{G \backslash e}\left(X^{\prime}\right)\right|+1 \leq 5$ holds which implies $\left|E_{G}\left(X^{\prime}\right)\right|=5$. Therefore $X^{\prime}=$ $\{x\},\{y\}, V(G)-\{x\}$, or $V(G)-\{y\}$.

From the graph $G$, delete the edge $e$ and split $x$. Let $H_{1}$ and $H_{2}$ be the two outcomes. Now we prove Lemma 7.2 by two cases: whether the case satisfies the assumption of Lemma 7.1 or not.

Case 1. If there are $K_{1}$ and $K_{2}$ such that for each $i=1,2,\left|E_{H_{i}}\left(K_{i}\right)\right|<5$ where $K_{i} \subseteq V\left(H_{i}\right)$ with $K_{i} \neq \emptyset,\{y\}, V\left(H_{i}\right)-\{y\}$, or $V\left(H_{i}\right)$. Then by Lemma 7.1, $G$ has a quadrilateral partition $\left(X_{1}, X_{2}, X_{3}, X_{4}\right)$, with center $x$ and width 2 . By symmetry, we may assume that $y \in X_{4}$, then for each $j=1,2,3,\left|E_{G}\left(X_{j}\right)\right|=\left|E_{G \backslash e}\left(X_{j}\right)\right|=5$. Since every 5-edge-cut is trivial, $X_{j}$ consists of exactly one vertex, say $x_{j}$.

Recall that in the assumption, $\mathcal{O}_{1}$ cannot be applied to an edge not incident with $z$. Let us focus on vertex $x_{2}$ and consider two cases: whether $x_{2}$ is $z$ or not.

If $x_{2} \neq z$, delete the edge $x x_{2}$ from $G$ and split $x_{2}$. Choose the outcome $H$ without a loop. $H$ can be obtained from $G \backslash x x_{2}$ by contracting the parallel edges $x_{1} x_{2}$. Thus $\left|E_{H}(X)\right| \geq 5$ for all $X \subseteq V(H)$ with $X \neq \emptyset,\{x\}, V(H)-$ $\{x\}$, or $V(H)$ since contraction does not change edge-connectivity.

Since edge $x x_{2}$ is not incident with $z, \mathcal{O}_{1}$ cannot be applied to this edge within $\mathcal{P}_{5}$. Splitting $x$ in the graph $H$ dose not result in a 5-edge-connected graph. By Lemma 4.2, $H$ has a quadrilateral partition $\left(X_{1}^{\prime}, X_{2}^{\prime}, X_{3}^{\prime}, X_{4}^{\prime}\right)$ with center $x$ and width 2 . By symmetry, we may assume that $x_{1} \in X_{1}^{\prime}$. H can be obtained from $G \backslash x x_{2}$ by contracting the parallel edges $x_{1} x_{2}$. Then for each $j=2,3,4,\left|E_{G}\left(X_{j}^{\prime}\right)\right|=\left|E_{H}\left(X_{j}^{\prime}\right)\right|=5$ since contraction does not change edge-connectivity, therefore $X_{j}^{\prime}$ consists of exactly one vertex. $H$ can be also obtained from $G \backslash x x_{2}$ by contracting the parallel edges $x_{2} x_{3}$, similar argument, $X_{1}^{\prime}$ consists of exactly one vertex. Consider the reverse operation, $G=W_{5}^{\prime}$.

If $x_{2}=z$, delete the edge $x x_{1}$ from $G$ and split $x_{1}$. Choose the outcome $H^{\prime}$ without a loop. Again by Lemma $4.2, H^{\prime}$ has a quadrilateral partition $\left(X_{1}^{\prime}, X_{2}^{\prime}, X_{3}^{\prime}, X_{4}^{\prime}\right)$ with center $x$ and width 2 . Say $x_{2} \in X_{4}^{\prime}$, then $H^{\prime}$ can be obtained from $G \backslash x x_{1}$ by contracting the parallel edges $x_{1} x_{2}$. By similar argument, for each $i=1,2,3, X_{i}^{\prime}$ consists of exactly one vertex. Since $z \notin X_{2}^{\prime}$, use the previous argument, we have $G=W_{5}^{\prime}$.

Case 2. Suppose that one of $H_{1}, H_{2}$, say $H_{1}$, satisfies $\left|E_{H_{1}}(X)\right| \geq 5$ for all $X \subseteq V\left(H_{1}\right)$ with $X \neq \emptyset,\{y\}, V\left(H_{1}\right)-\{y\}$, or $V\left(H_{1}\right)$. Remind that $H_{1}$ is obtained from $G$ by deleting $x y$ and splitting $x$. Since $\mathcal{O}_{1}$ cannot be applied to the edge $e=x y$ that is not incident with $z$ in $G$ within $\mathcal{P}_{5}$, splitting $y$ in $H_{1}$ does not result in a 5-edge-connected graph. By Lemma 4.2, $H_{1}$ has a quadrilateral partition $\left(Y_{1}, Y_{2}, Y_{3}, Y_{4}\right)$ with center $y$ and width 2 . Let us 
focus on graph $G$. If $x$ is incident with three of the four partitions, then $G$ contains a $K_{5}$-minor, which implies that $G$ is not planar. If $x$ is incident with two non-adjacent partitions, say $Y_{1}$ and $Y_{3}$, then $G$ contains a $K_{3,3^{-}}$ minor, which again implies that $G$ is not planar. Therefore, $x$ can be only incident to either one partition or two adjacent partitions. Without loss of generality, we may assume that $x$ is not incident with $Y_{3}, Y_{4}$, then for each $j=3,4,\left|E_{G}\left(Y_{j}\right)\right|=\left|E_{H_{1}}\left(Y_{j}\right)\right|=5$. Therefore, $Y_{j}$ consists of exactly one vertex, say $y_{j}$. Suppose $y_{4} \neq z$ (otherwise, $y_{3} \neq z$ ). Then in the graph $G$, delete the edge $y y_{4}$ and split $y_{4}$. Choose the outcome $H^{\prime}$ without a loop. The graph $H^{\prime}$ can be obtained from $G \backslash y y_{4}$ by contracting the parallel edges $y_{3} y_{4}$. Since contraction dose not change edge-connectivity, $\left|E_{H^{\prime}}(X)\right| \geq 5$ for all $X \subseteq V\left(H^{\prime}\right)$ with $X \neq \emptyset,\{y\}, V\left(H^{\prime}\right)-\{y\}$, or $V\left(H^{\prime}\right)$. By Lemma 4.2, $H^{\prime}$ has a quadrilateral partition $\left(Y_{1}^{\prime}, Y_{2}^{\prime}, Y_{3}^{\prime}, Y_{4}^{\prime}\right)$ with center $y$ and width 2 because $y_{4} \neq z$. By symmetry, we may assume that $y_{3} \in Y_{4}^{\prime}$. Since contraction does not change edge-connectivity, we can again prove that for each $i=1,2,3, Y_{i}^{\prime}$ consists of exactly one vertex. Using the same argument as in case 1 to obtain $G=W_{5}^{\prime}$.

Proof of Theorem 7.1 We prove by induction on $|V(G)|$.

Recall that $\mathcal{O}_{1}$ reduces vertices by two, $\mathcal{O}_{11}$ and $\mathcal{O}_{12}$ reduce vertices by four, and $G$ has an even number of vertices. We need to prove that the result holds for both $|V(G)|=2$ and $|V(G)|=4$ in the basic case.

If $|V(G)|=2$, then $G=5 K_{2}$ and thus the result holds.

If $|V(G)|=4$, there is only one case $\left\{x_{1} x_{2}, x_{1} x_{2}, x_{2} x_{3}, x_{2} x_{3}, x_{3} x_{4}, x_{3} x_{4}\right.$, $\left.x_{4} x_{1}, x_{4} x_{1}, x_{1} x_{3}, x_{2} x_{4}\right\}$ where $\left\{x_{1}, x_{2}, x_{3}, x_{4}\right\}$ are the vertices and $\mathcal{O}_{1}$ can be applied within $\mathcal{P}_{5}$. The result holds in this case.

Next, we consider the general case when $|V(G)|>4$. Since the result of applying each $\mathcal{O}_{i}(i=1,11,12)$ is always 5 -regular and planar, we only need to show that

(*) $G$ can be reduced to a 5-edge-connected graph by applying $\mathcal{O}_{1}, \mathcal{O}_{11}$, or $\mathcal{O}_{12}$ once.

If $\mathcal{O}_{1}$ can be applied within $\mathcal{P}_{5}$, then $\left(^{*}\right)$ holds. Suppose $\mathcal{O}_{1}$ cannot be applied. If every 5-edge-cut is trivial, then by Lemma 7.2, $G=W_{5}^{\prime}$. Therefore, $\mathcal{O}_{11}$ or $\mathcal{O}_{12}$ can be applied, $(*)$ is proved.

Suppose that $\mathcal{O}_{1}$ cannot be applied and there is a non-trivial 5-edgecut $F$. Let $X, Y$ be the two components of $G \backslash F$. Then $|V(X)| \geq 2$ and $|V(Y)| \geq 2$. Choose a 5-edge-cut and $X$ such that $X$ does not contains any non-trivial 5-edge-cut. Contract $Y$ into a vertex $z$ in $G$. Let $K$ be the outcome. Then $K \in \mathcal{P}_{5}$ since contraction dose not change edge-connectivity. 
Now we will prove that $\mathcal{O}_{1}$ cannot be applied to $K$ within $\mathcal{P}_{5}$ to a simple edge that is not incident with $z$. Assume that this is not true. Then there exists a simple edge $e=x_{1} x_{2}$ with $x_{1}, x_{2} \in V(X)$ such that applying $\mathcal{O}_{1}$ to $e$ results in a graph $K_{1} \in \mathcal{P}_{5}$. Do the same operation on the graph $G$, let $G_{1}$ be the corresponding outcome. We will prove that $G_{1} \in \mathcal{P}_{5}$. To prove this, we need to prove $\left|E_{G_{1}}(Z)\right|=m_{G_{1}}(Z) \geq 5$ for all $Z \neq \emptyset, V\left(G_{1}\right)$ where $m$ is a notation from chapter 4 .

Choose any vertex set $Z \subseteq V\left(G_{1}\right)$ with $Z \neq \emptyset, V\left(G_{1}\right)$. Let $Y_{1}=Z \cap$ $V(Y), Y_{2}=V\left(G_{1} \backslash Z\right) \cap Y, X_{1}=Z \cap V\left(G_{1} \backslash Y\right), X_{2}=V\left(G_{1} \backslash Z\right) \cap V\left(G_{1} \backslash Y\right)$.

First, we prove that if one of $X_{1}, X_{2}, Y_{1}, Y_{2}$ is empty, then $m_{G_{1}}(Z) \geq 5$.

For each $i=1,2$,

if $Y_{i}=\emptyset$, then $m_{G_{1}}(Z)=m_{G_{1}}\left(X_{i}\right)=m_{K_{1}}\left(X_{i}\right) \geq 5$ since $K_{1} \in \mathcal{P}_{5}$;

if $X_{i}=\emptyset$, then $m_{G_{1}}(Z)=m_{G_{1}}\left(Y_{i}\right)=m_{G}\left(Y_{i}\right) \geq 5$. since $G \in \mathcal{P}_{5}$.

Now we prove that $m_{G_{1}}(Z) \geq 5$ holds if all the four vertex sets are non-empty.

Suppose that $X_{1}, X_{2}, Y_{1}, Y_{2}$ are all non-empty.

$$
m_{G}\left(Y_{1}, X\right)+m_{G}\left(Y_{2}, X\right)=m_{G}(X)=5,
$$

thus one of $m_{G}\left(Y_{1}, X\right) \leq 2$ and $m_{G}\left(Y_{2}, X\right) \leq 2$ holds, say $m_{G}\left(Y_{1}, X\right) \leq 2$.

$$
m_{G}\left(Y_{1}, Y_{2}\right)+m_{G}\left(Y_{1}, X\right)=m_{G}\left(Y_{1}\right) \geq 5
$$

Thus, $m_{G_{1}}\left(Y_{1}, Y_{2}\right)=m_{G}\left(Y_{1}, Y_{2}\right) \geq 5-m_{G}\left(Y_{1}, X\right) \geq 5-2=3$

$$
m_{K_{1}}\left(X_{1},\{z\}\right)+m_{K_{1}}\left(X_{2},\{z\}\right)=m_{K_{1}}(\{z\})=5,
$$

similarly, we may assume that $m_{K_{1}}\left(X_{1},\{z\}\right) \leq 2$

$$
m_{K_{1}}\left(X_{1}, X_{2}\right)+m_{K_{1}}\left(X_{1},\{z\}\right)=m_{K_{1}}\left(X_{1}\right) \geq 5
$$

Thus, $m_{G_{1}}\left(X_{1}, X_{2}\right)=m_{K_{1}}\left(X_{1}, X_{2}\right) \geq 5-m_{K_{1}}\left(X_{1},\{z\}\right) \geq 5-2=3$

Therefore, $m_{G_{1}}(Z) \geq m_{G_{1}}\left(X_{1}, X_{2}\right)+m_{G_{1}}\left(Y_{1}, Y_{2}\right) \geq 3+3 \geq 5$

Now we have already exhausted all the cases and proved that $\left|E_{G_{1}}(Z)\right|=$ $m_{G_{1}}(Z) \geq 5$ holds for all $Z \subseteq G_{1}$ with $Z \neq \emptyset, V\left(G_{1}\right)$. Therefore, $G_{1} \in \mathcal{P}_{5}$, which contradicts with our assumption. That is, $\mathcal{O}_{1}$ cannot be applied in $K$ within $\mathcal{P}_{5}$ to a simple edge that is not incident with $z$.

Since $K \in \mathcal{P}_{5}, K \neq 5 K_{2}$, and every 5 -edge-cut in $K$ is trivial. $\mathcal{O}_{1}$ cannot be applied within $\mathcal{P}_{5}$ to a simple edge that is not incident with $z$. By Lemma $7.2, K=W_{5}^{\prime}$. If $z$ is a vertex incident with 5 simple edges, then $\mathcal{O}_{11}$ can be applied to $G .\left(^{*}\right)$ is proved. Otherwise, $\mathcal{O}_{12}$ can be applied to $G .\left(^{*}\right)$ is proved again. 


\section{Further Remarks}

For $k=1,2,3,4,5$, we proved generating theorems for the classes $\mathcal{P}_{k}$ of all $k$-edge-connected 5-regular planar graphs. One of next natural questions is what would happen if we added another restriction, for example, girth. The girth of a graph $G$, denoted by $g$, is the minimum length of cycles contained in $G$. Let us define $\mathcal{P}_{k, g}$ as the subclass of $\mathcal{P}_{k}$ such that every graph in $\mathcal{P}_{k, g}$ has a girth at least $g$. Since $\mathcal{P}_{k, 1}=\mathcal{P}_{k}$ holds for any $k$, and if $k>3$, then $\mathcal{P}_{k, 2}=\mathcal{P}_{k}$ holds, problems we interested are to prove generating theorems for $\mathcal{P}_{k, g}$ with $g>1$ and $k=0,1,2,3$. The following are our natural conjectures for $g=2$.

Conjecture 8.1 Every graph in $\mathcal{P}_{k, 2}$ with $k=0,1,2$ can be reduced within $\mathcal{P}_{k, 2}$ by $\mathcal{O}_{1}, \mathcal{O}_{3}$, and $\mathcal{O}_{6}$ to $5 K_{2}$.

Let $P^{\prime}$ be the graph obtained from the prism $P$ by replacing each edge of a triangle in $P$ by two parallel edges. As a result, the graph $P^{\prime}$ consists of six vertices, twelve multiple edges and three simple edges.

Conjecture 8.2 Every graph in $\mathcal{P}_{3,2}$ can be reduced within $\mathcal{P}_{3,2}$ by $\mathcal{O}_{1}$ and $\mathrm{O}_{3}$ to $P^{\prime}$ or $5 K_{2}$.

If we move to $g=3$, we deal with 5 -regular simple planar graphs and need to change graph operations since $\mathcal{O}_{1}$ often provides inevitable multiple edges, for example, if an applied edge is a spoke of $W_{5}$. See [8] for a study in this direction.

Another natural research expansion is to pursue splitter theorems for $\mathcal{P}_{k}$ and $\mathcal{P}_{k, g}$. Suppose a graph $G$ "contains" another graph $H$. Then how can $G$ be built up from $H$ in such a way that certain properties of $G$ and $H$ are preserved during the construction process? Probably the best-known result to answer this kind of question is the one by Seymour [13], for general matroids, and Negami [11], for graphs only, which explains the construction when the containment relation is the minor relation and the property to preserve is 3-connectedness. These answers are known as splitter theorems. Ding and Kanno have proved splitter theorems for several classes of 3 -regular, 4-regular or 4-regular planar graphs (see $[5,6,7]$ ).

\section{References}

[1] D. Barnette, On generating planar graphs, Discrete Mathematics 7 (1977), 199-208. 
[2] D. Barnette, Generating the $c^{*} 5$-connected graphs, Israel Journal of Mathematics 28 (1977), Nos. 1-2, 151-160.

[3] H. J. Broersma, A. J. W. Duijvestijn, and F. Göbel, Generating all 3-connected 4-regular planar graphs from the graph of the octahedron graph, J. Graph Theory 17 (1993), No.5, 613-620.

[4] J. W. Butler, A generation procedure for the simple 3-polytopes with cyclically 5-connected graphs, Canadian Journal of Mathematics XXVI (1974), No.3, 686-708.

[5] G. Ding and J. Kanno, Splitter Theorems for cubic graphs, Combin. Probab. Comput.15 No.3 (2006), 355-375.

[6] G. Ding and J. Kanno, Splitter Theorems for 4-regular graphs, submitted.

[7] J. Kanno, Splitter theorems for 3- and 4-regular graphs, Ph.D. dissertation, Louisiana State University, Baton Rouge, Louisiana, 2003. http://etd.lsu.edu:8085/docs/available/etd-0529103-123537/

[8] J. Kanno and M. Kriesell, A generating theorem for 5-regular simple planar graphs I, Congressus Numerantium 185 (2007), 127-143.

[9] J. Lehel, Generating all 4-regular planar graphs from the graph of the octahedron, J. Graph Theory 5 (1981), No.4, 423-426.

[10] P. Manca, Generating all planar graphs of degree four, J. Graph Theory 3 (1979), 357-364.

[11] S. Negami, A characterization of 3-connected graphs containing a given graph, J. Combinatorial Theory Series B, 32 (1982), 69-74.

[12] O. Ore, The four-color problem, Academic Press, New York, 1967.

[13] P. D. Seymour, Decomposition of regular matroids, J. Combinatorial Theory Series B, 28 (1980), 305-359.

[14] A. Schrijver, Combinatorial Optimization: Polyhedra and Efficiency, Springer, Berlin, 2003

[15] E. Steinitz and H. Rademacher, Vorlesungen über die Theorie de Polyeder, Berlin 1934. 